\title{
Carbon Dioxide Injection for Hypervelocity Boundary Layer Stability
}

\author{
Ross M. Wagnild ${ }^{1}$ and Graham V. Candler ${ }^{2}$ \\ University of Minnesota, Minneapolis, Minnesota, 55455 \\ Ivett A. Leyva ${ }^{3}$ \\ Air Force Research Laboratory, Edwards AFB, California, 93536 \\ Joe S. Jewell ${ }^{4}$ and Hans G. Hornung ${ }^{5}$ \\ Caltech, Pasadena, California, 91125
}

\begin{abstract}
An experimental method for introducing carbon dioxide as a means of stabilizing a hypervelocity boundary layer over a slender bodied vehicle is investigated through the use of numerical simulations. In the current study, two different test bodies are examined. The first is a five-degree-half-angle cone currently under research at the T5 Shock Tunnel with a four cm porous wall insert used to transpire gas into the boundary layer. The second test body is a similar cone with a porous wall over a majority of the cone surface. Computationally, the transpiration is performed using an axi-symmetric flow simulation with wall-normal blowing. The effect of the injection and the transition location are gauged by solving the parabolized stability equations and using the semi-empirical $\mathrm{e}^{\mathrm{N}}$ method. The results show transition due to the injection for the first test body and a delay in the transition location for the second test body as compared to a cone without injection under the same free-stream conditions. The mechanism for the stabilizing effect of carbon dioxide is also explored through selectively applying non-equilibrium processes to the stability analysis. The results show that vibrational non-equilibrium plays a role in reducing disturbance amplification, however, other factors also contribute.
\end{abstract}

\section{Introduction}

$I^{\prime}$

n the study of hypersonic vehicles, when a boundary layer transitions from laminar to turbulent, both the skin Ifriction and surface heat transfer rate on the vehicle increase in magnitude. The implications for high speed vehicle design are increased drag and greater thermal protection thickness, which require a greater vehicle weight and result in a reduction of fuel efficiency.

The purpose of the current study is to further investigate the stabilizing effect of carbon dioxide on hypervelocity boundary layers. The concept for this work is derived from combining three findings from past research. First, transition on slender bodies in hypervelocity flows is caused by amplification of acoustic waves trapped in the boundary layer. Second, non-equilibrium effects such as molecular vibration and dissociation can damp acoustic waves. Lastly, carbon dioxide has been found to be well suited to absorb acoustic energy in an enthalpy range relevant to realistic applications. These findings point to the beneficial application of adding carbon dioxide into a boundary layer in order to delay transition onset. Experimentally, this is attempted by introducing carbon dioxide into the boundary layer by transpiring it through a porous material. The experiments are being performed on a fivedegree-half-angle cone in the Graduate Aeronautical Laboratories at California Institute of Technology (GALCIT) Free-Piston Shock Tunnel, T5. A computational fluid dynamics (CFD) simulation that solves the reacting,

\footnotetext{
${ }^{1}$ Graduate Student, University of Minnesota, Minneapolis, Minnesota, AIAA Student Member.

${ }^{2}$ Professor, University of Minnesota, Minneapolis, Minnesota, AIAA Fellow.

${ }^{3}$ Lead, Combustion Devices Group, AFRL, Edwards AFB, California, AIAA Senior Member,

${ }^{4}$ Graduate Student, Caltech, Pasadena, California, AIAA Student Member.

${ }^{5}$ Professor Emeritus, Caltech, Pasadena, California, AIAA Fellow.
} 
compressible Navier-Stokes equations is used to recreate the experiment. The simulations are solved such that the boundary layer is completely laminar. These data are then used to provide the mean flow to be analyzed with the software suite STABL (Stability and Transition Analysis for hypersonic Boundary Layers), which solves the linear set of parabolized stability equations. A cone without injection is also studied in order to calibrate this method to the T5 tunnel environment and to form a baseline for determining transition delay. A similar study is performed on a theoretical cone with a longer interval of transpiration in order to isolate non-equilibrium effects. The objective is to show that adding carbon dioxide delays transition on the test body. This work leads to further analysis on variations on the design to gain insight into using chemical reactions and vibrational degrees of freedom to stabilize boundary layers. This research is part of a continuing effort to actively control boundary layer transition by means of $\mathrm{CO}_{2}$ gas injection, which, to the authors' knowledge is a new technology.

\section{Background}

A summary of the theoretical basis of this research is laid out by Leyva et al. ${ }^{1}$, however, a brief review of the essential concepts follows. For slender bodies in hypervelocity flow, transition occurs due to second mode disturbances, also known as a Mack mode, which is an inviscid effect caused by acoustic waves trapped in the boundary layer. Once these disturbances are sufficiently amplified, the flow in the boundary layer transitions from laminar to turbulent. Hornung et al. ${ }^{2}$ summarizes the work that has been done to characterize transition on slender bodies using the GALCIT Free-Piston Shock Tunnel, T5. Hornung's study begins with the measurement of transition on a five-degree-half-angle cone with free-stream compositions of nitrogen, air, and carbon dioxide. Their results show that carbon dioxide had higher transition Reynolds' number as compared to that of nitrogen and air. However, this difference was not seen in experiments at lower total enthalpies. Hornung attributes the difference in transition behavior at different enthalpies to the acoustic damping of non-equilibrium processes such as molecular vibration and dissociation.

The effect of non-equilibrium has also been studied using numerical simulations as a means for delaying transition. Analyses, such as that of Johnson et al. ${ }^{3}$, prove that adding chemical and thermal non-equilibria to a simulation increase the stability of a boundary layer. This increase in stability is due to endothermic reactions and vibrational modes absorbing the energy of the disturbances. In addition, these analyses find that the stabilizing effect is greater when the fluid consists of carbon dioxide rather than air, which is a result of various properties of carbon dioxide. First, carbon dioxide has a relatively low dissociation temperature compared to that of diatomic oxygen and nitrogen. Second, carbon dioxide has three vibrational modes, whereas oxygen and nitrogen have only one. Lastly, carbon dioxide most efficiently absorbs acoustic energy at frequencies similar to those that cause transition for high Mach numbers.

A more in-depth analysis of these mechanics was done by Fujii and Hornung ${ }^{4}$, which found that carbon dioxide most efficiently absorbs acoustic frequencies in the same range as those that cause transition, typically around 1-10 $\mathrm{MHz}$, displayed in Figure 1. In general, Carbon dioxide most efficiently absorbs acoustic energy in this frequency range near $2000 \mathrm{~K}$, as seen in Figure 2. Fujii also notes that when two peaks are present in the absorption rate versus frequency curve they correspond to the type of relaxation. The first peak, at a lower frequency, is due to dissociation, and the second peak, at a higher frequency, is due to vibrational relaxation. Fujii's data show that vibrational relaxation will be the dominant mechanism for disturbance energy absorption for the current study. As the temperature increases, more carbon dioxide dissociates, resulting in fewer molecular vibrators and thus a weaker peak in the vibrational absorption rate. However, if the temperature is sufficiently high, the chemical relaxation can begin to have significant acoustic energy absorption. 


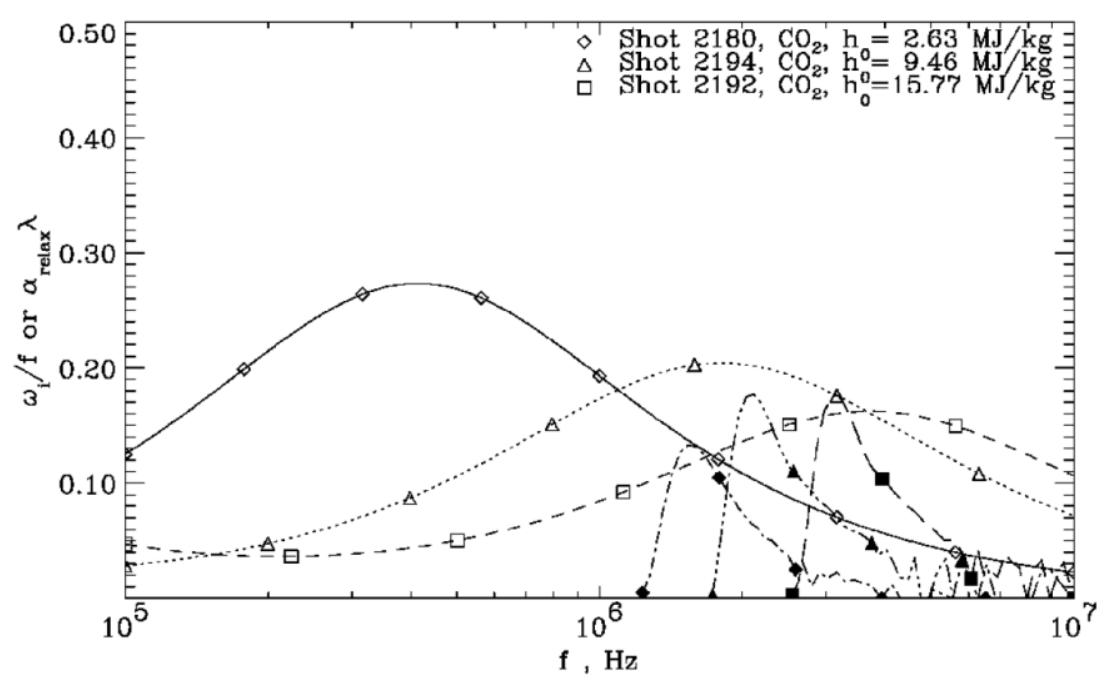

Figure 1. This plot compares the disturbance frequencies calculated using a linear stability analysis against the absorption rate of carbon dioxide on a 45-degree, swept cylinder. The disturbance frequencies are in the range of 1-10 MHz, which is typical for the second mode. This plot is taken from Fujii and Hornung ${ }^{4}$.

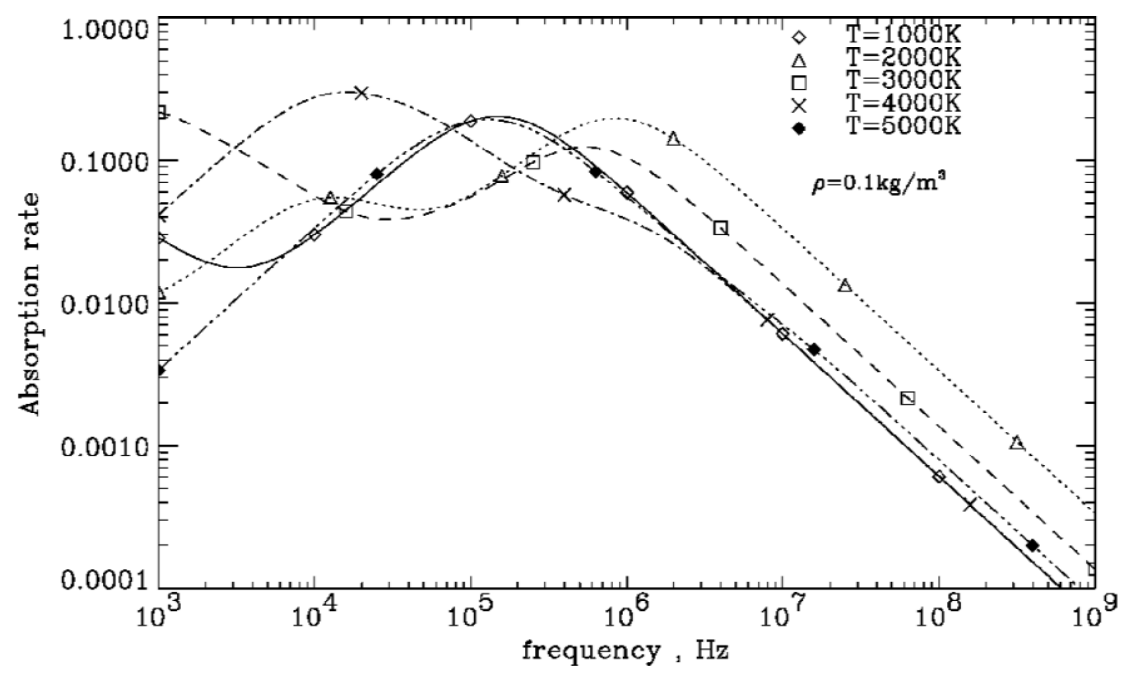

Figure 2. Sound absorption rate based on frequency for carbon dioxide at various temperatures. Carbon dioxide absorbs disturbance energy most effectively at $2000 \mathrm{~K}$ for the range of 1-10 MHz. This plot is taken from Fujii and Hornung ${ }^{4}$.

The previous work by Leyva et al. ${ }^{1}$ included a preliminary study to test the feasibility of injecting carbon dioxide to delay boundary layer transition by altering the free-stream mixture. As inferred from Figure 3, increasing the mole fraction of carbon dioxide increases the transition Reynolds' number, reinforcing the findings of Hornung et al. ${ }^{2}$ These tests could be considered the result from an optimal addition, mixing, and heating of carbon dioxide in a free-stream composed of air or nitrogen. Though the tests with air as the primary free-stream component are limited, a mole fraction of fourteen percent would have a noticeable delay in transition. 


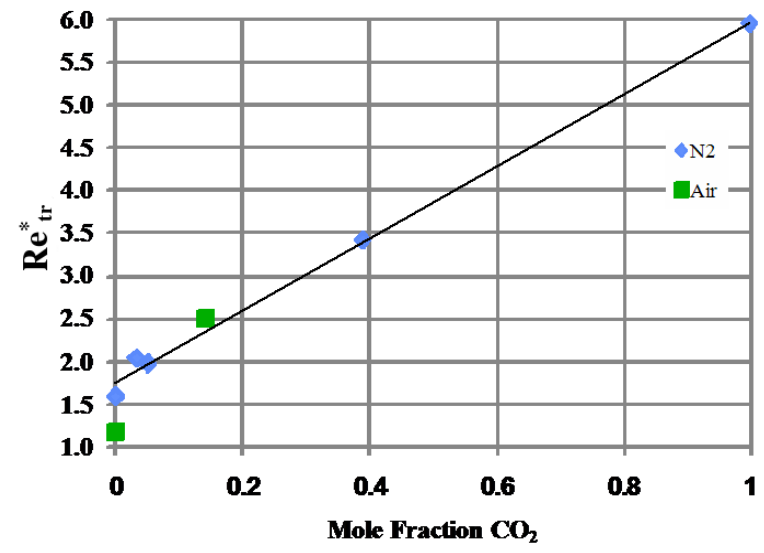

Figure 3. This plot summarizes preliminary work performed at the T5 tunnel. An increase in the amount of carbon dioxide delays transition further down the cone. This plot is taken from Leyva et al. ${ }^{1}$

Over the last year the models proposed in Leyva et al. ${ }^{1}$ were tested more thoroughly experimentally and numerically. These models include a number of rows, ranging from one to four, of evenly spaced injector ports at a shallow angle to surface. The objective of this low angle injection was to add carbon dioxide while minimizing the disturbance caused to the boundary layer, with regards to the penetration height and the loss of stream-wise momentum. At the T5 tunnel, experiments revealed that the flow was transitioning at the location of injection, indicating that the injection was causing too large of a disturbance to the boundary layer. Further testing also showed that the presence of the ports alone, no gas injection, was causing a disturbance large enough to transition the boundary layer, seen in Figure 4. Numerical testing of a simplified model of the experiment showed promise, however, upon completion of a grid convergence study, it was found that the shear layer that develops between the shock layer and jet gas became unstable and began to breakdown, shown in Figure 5. Due to the tendency of the discrete jets to cause transition, a model using an axi-symmetric transpiration was built and is currently under research.
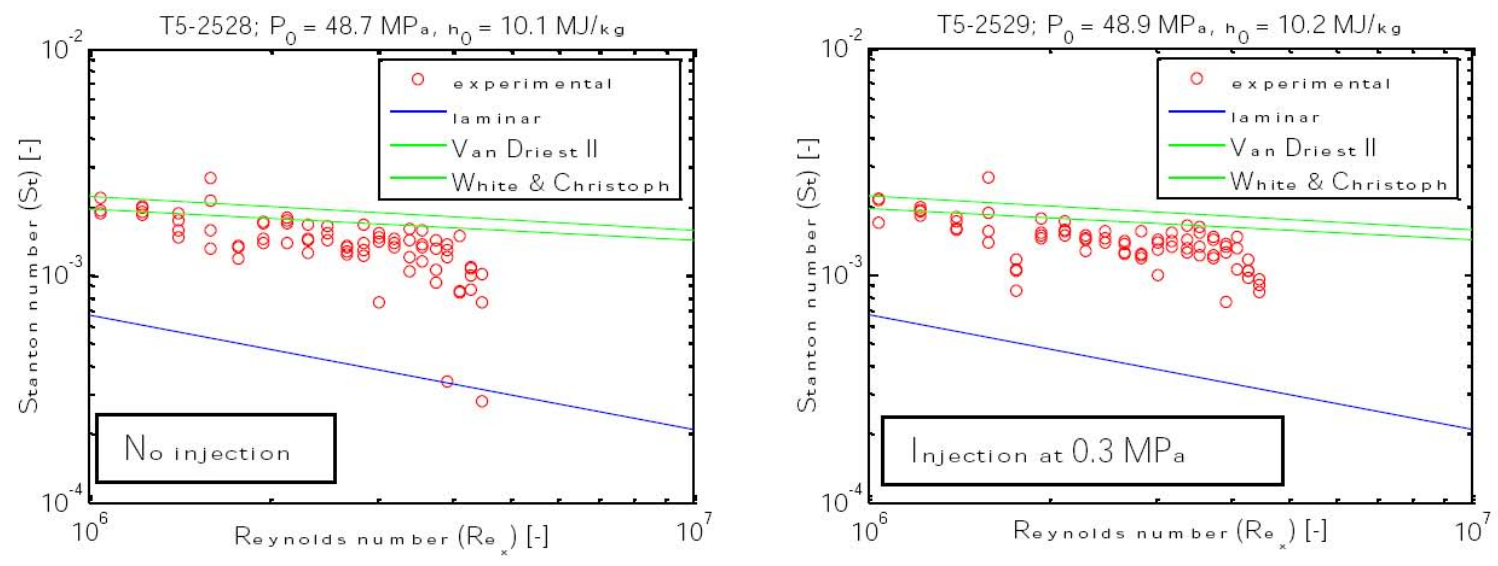

Figure 4. These plots are data from the thermocouples for two shots at the T5 tunnel. Both shots have four rows of discrete injector ports and both show fully turbulent heating. This plot is taken from Leyva et al. ${ }^{5}$ 


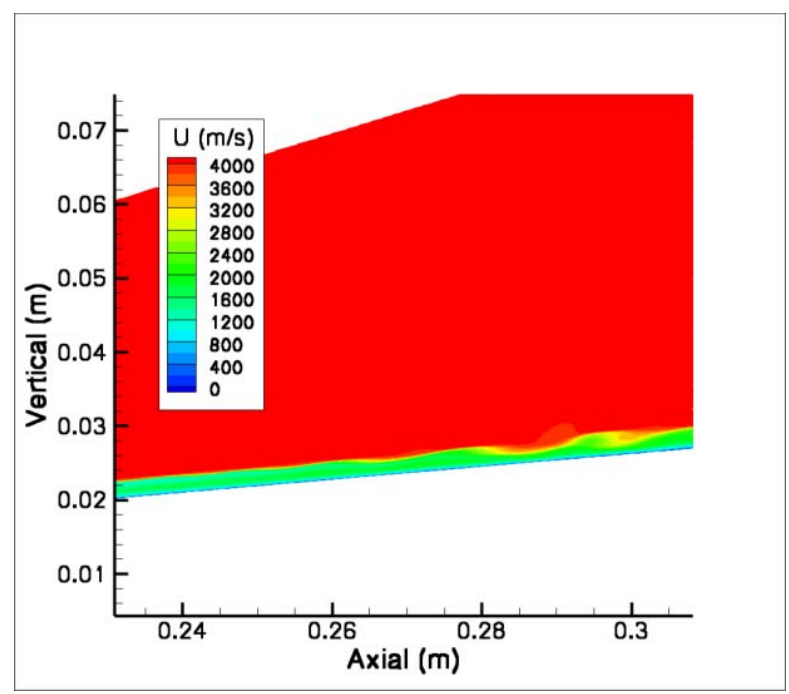

Figure 5. This plot shows contours of stream-wise velocity from a simulation of carbon dioxide injection through a discrete port at $0.58 \mathrm{MPa}$. The injection resulted in shear-layer breakdown.

\section{Computation Model}

Due to the axi-symmetric nature of the injection scheme, the axi-symmetric, reacting, compressible NavierStokes equations are solved using the structured solver DPLR ${ }^{6}$. The solutions are completely laminar and provide a mean flow for the stability analysis. The inviscid fluxes are evaluated using the second-order accurate, modified Steger-Warming flux vector splitting method. The viscous fluxes are calculated using a second -order central method. The time integration is the first-order accurate, implicit, line-relaxation method. Solutions are calculated in parallel using MPI to handle inter-processor communication. This solver is contained in the software suite called STABL, written by Dr. Heath Johnson.

The stability analysis is performed with the PSE-Chem solver, also a part of STABL. PSE-Chem solves the twodimensional linear parabolized stability equations, which are derived from the Navier-Stokes equations. The PSEChem solver also includes the effects of finite-rate chemical reactions and translational-vibrational energy exchange. The transition prediction is based on the semi-empirical $\mathrm{e}^{\mathrm{N}}$ method. This method uses experimentally measured transition locations to estimate where the calculated disturbance growth rate will cause transition. For a more detailed explanation of the stability analysis, the reader is referred to the works by Johnson and Candler ${ }^{7}$ and Johnson et al. ${ }^{8}$.

These analyses are performed using a seven species chemistry set which includes $\mathrm{CO} 2, \mathrm{CO}, \mathrm{N} 2, \mathrm{O} 2, \mathrm{NO}, \mathrm{N}$, and O. For simulations involving the injection of nitrogen or air, a simplified version of this set including only N2, O2, $\mathrm{NO}, \mathrm{N}$, and $\mathrm{O}$ is used. A species' viscosity is calculated using a composition of various models, each appropriate for a different temperature range. The models and unified using blending functions to maintain continuity for both the value and derivative value of viscosity. The conductivity is calculated using Eucken's relation. The mixture quantities are then calculated using Wilke's semi-empirical mixing law. It is assumed that vibrational-vibrational energy exchanges happen on a relatively short time scale and thus are in equilibrium and governed by a single temperature, $\mathrm{T}_{\mathrm{v}}$. It is also assumed that rotation and translation are coupled and are governed by the translational temperature. The translational-vibrational energy exchanges are governed by the Laudau-Teller model. The characteristic relaxation times are based on the Millikan and White model, except for the relaxation times involving $\mathrm{CO} 2$, which are based on those determined by Camac ${ }^{9}$. The chemical reactions used for these simulations are given in Table 3 in the appendix. The forward reaction rate coefficients are taken from Park et al. ${ }^{10}$, except reactions five and six, which are taken from Bose and Candler ${ }^{11,12}$, respectively. The equilibrium coefficients are taken from Park $^{13}$, except for reactions one and seven, which result from fitting the data given by McBride et al. ${ }^{14}$. 


\section{T5 Tunnel Test Body}

The T5 tunnel test body is a sharp, five-degree-half-angle cone. The surface of the cone is smooth with the exception of a porous material between $12.8 \mathrm{~cm}$ and $16.9 \mathrm{~cm}$ from the nose of the cone. This material has pores with a diameter of approximately $10 \mu \mathrm{m}$. In the numerical simulations, the porous wall transpiration is modeled through the use of the wall-blowing condition contained in the DPLR solver. It is assumed that mass flow rate through the porous material is a constant over a given area. In an axi-symmetric simulation, this results in a top hat distribution across the length of the injection region. To maintain continuity of the mass flux through the wall between the solid and porous surfaces, a function based on hyperbolic tangent is generated to approximate the top hat distribution.

The grid used in these simulations is generated by a module included in STABL. Due to the structured nature of the DPLR solver, all cells of the grid are quadrilateral. The wall-normal direction has 300 cells and the cells are clustered near the wall to guarantee that the $\mathrm{y}^{+}$values are all less than one and have a growth rate of 2.7 percent in order to properly resolve the boundary layer gradients. The wall-tangential direction has 1249 total cells. A majority of these cells are clustered near the nose and have a maximum growth rate of 5.8 percent until the injection region. At this location, 100 cells are distributed across the porous wall surface. From the end of the injection region until the end of the cone the cell sizes have a maximum growth rate of 2.4 percent.

The conditions for the current simulations are chosen to be similar to shots performed in the T5 tunnel and consist of a partially dissociated mixture of air with the physical properties listed in Table 1 as both the reservoir for the tunnel and the free-stream. The free-stream Mach number is approximately 5.3. The wall condition for this experiment is considered to be isothermal with a temperature of $293 \mathrm{~K}$.

Table 1. Free-stream Conditions Used for the T5 Tunnel Simulations.

\begin{tabular}{|c|c|c|c|}
\hline \multicolumn{2}{|c|}{ Stagnation Conditions } & \multicolumn{2}{c|}{ Free-stream Conditions } \\
\hline Pressure $(\mathrm{MPa})$ & 50.92 & Density $\left(\mathrm{kg} / \mathrm{m}^{3}\right)$ & 0.05572 \\
\hline Temperature $(\mathrm{K})$ & 5968.5 & Temperature $(\mathrm{K})$ & 1369.4 \\
\hline Entalpy $(\mathrm{MJ} / \mathrm{kg})$ & 9.51 & Velocity $(\mathrm{m} / \mathrm{s})$ & 3957.9 \\
\hline
\end{tabular}

A number of cases are run using carbon dioxide as the injection gas with a total mass flow rate ranging from 3 grams per second $(\mathrm{g} / \mathrm{s})$ to $13.5 \mathrm{~g} / \mathrm{s}$. Air and nitrogen are also injected to determine the effectiveness of injection gases with different vibrational and chemical parameters. For these cases the injection gas temperature is assumed to be equal to the wall temperature. To gauge the effect of heating the carbon dioxide before injection, four cases are run with injection temperatures of 1000, 1500, 2000, and $2500 \mathrm{~K}$. These last six cases all have different mass flow rates in order to match the penetration height of transpiration for the cold carbon dioxide case with a mass flow rate of $13.5 \mathrm{~g} / \mathrm{s}$. A summary of these test cases is provided in Table 4 in the appendix.

\section{A. Smooth Cone}

As previously mentioned, the semi-empirical $\mathrm{e}^{\mathrm{N}}$ method requires calibration to the environment of the test facility to determine the growth rate that will cause transition. A stability analysis is performed on a cone without injection to provide this calibration and also to serve as a baseline from which a potential delay in transition will be measured. The experimental data are taken from the shot 2540 and the comparison of these and the computational data are seen in Figure 6a. Transition onset occurs at approximately $62.5 \mathrm{~cm}$ along the cone's surface, which corresponds to an $\mathrm{N}$ factor of approximately 9.47. This differs from previously published data $^{3}$ and requires more thorough investigation to ensure the accuracy of this result for the T5 facility. However, this is the $\mathrm{N}$ factor that will be used for the current study. The accuracy of the mean flow can also be gauged from Figure 6a by the similarity in the laminar heating rates on the cone surface.

The stability diagram produced by the LST analysis, seen in Figure 6b, plots contours of amplification over a range of frequencies and distances along the cone. The $1^{\text {st }}, 2^{\text {nd }}$, and $3^{\text {rd }}$ mode estimates are plotted as well as the most-amplified frequency. The diagram shows that the disturbances that cause transition are second mode, demonstrating that this case is well-suited to test the injection of carbon dioxide. 


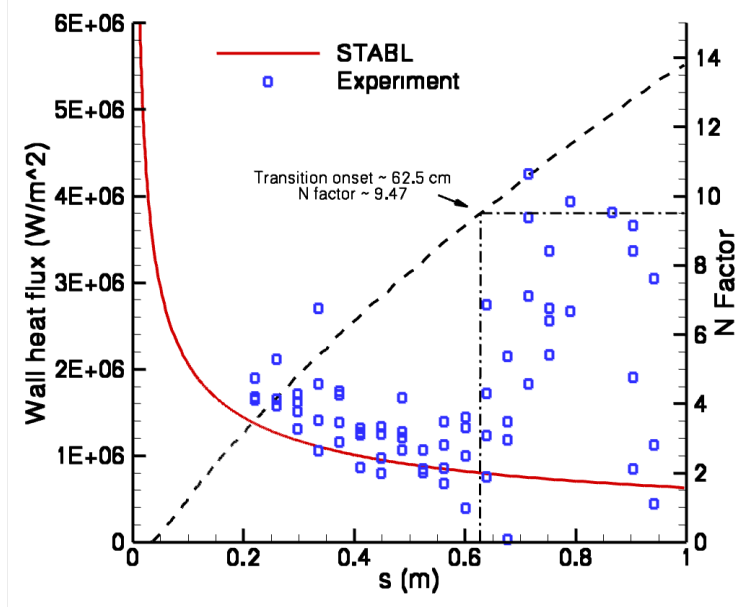

a) Plot of Heat Flux and $\mathbf{N}$ factor

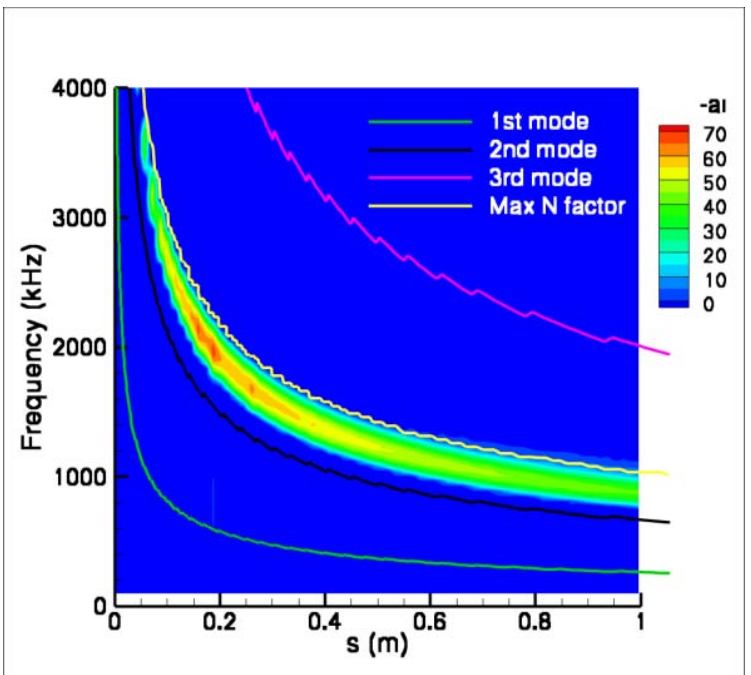

b) LST Stability Diagram

Figure 6. These plots show the results from the stability analysis of a smooth cone. The plot on the left, a), shows the comparison of heat flux between the simulation and experiment. Transition onset is gauged by the rapid increase in heat flux and the $\mathrm{N}$ factor at that location is measured. The plot on the right, b), is the LST stability diagram, disturbance mode frequency estimates, and the most-amplified frequency.

\section{B. Cold Carbon Dioxide Injection}

Traditionally, wall-blowing has a destabilizing effect on a boundary layer, so it is expected that a higher mass flow rate of blowing would cause a decrease in stability. The stability analyses of cases with injection of $3 \mathrm{~g} / \mathrm{s}$ through $7.5 \mathrm{~g} / \mathrm{s}$ follow this trend, seen in Figure 7a. All of these cases show sufficient amplification to transition immediately following the injection region. The $\mathrm{N}$ factor plots for cases of $6 \mathrm{~g} / \mathrm{s}$ and $7.5 \mathrm{~g} / \mathrm{s}$ are truncated toward the nose of the vehicle due the presence of a recirculation zone at the front of the transpiration. It is unknown if this recirculation exists in the experimental flow field and also the effect the recirculation has on the boundary layer stability. Previous experience has cast doubt on the accuracy of a PSE analysis through a recirculation zone and therefore it has been omitted from the results. All of the $\mathrm{N}$ factor profiles are similar in the region immediately following injection, indicating that the flow features such as the penetration height of the injection and distance of the boundary layer recovery are also similar, with their amplitudes dependent on the total mass injected.

For the cases with injection of $10 \mathrm{~g} / \mathrm{s}$ to $12.0 \mathrm{~g} / \mathrm{s}$, the stability analyses show a further increase in amplification with injection rate, seen in Figure 7b. The cases with injection of $13.0 \mathrm{~g} / \mathrm{s}$ and $13.5 \mathrm{~g} / \mathrm{s}$ show an increase in stability, however, all cases in this range have sufficient amplification after the injection to transition.

One feature common to all injection cases simulated is the growth of $\mathrm{N}$ factor more rapid than in the smooth cone case on the later part of the cone. The reason for this is best demonstrated by the LST stability diagram from the case with injection of $6.0 \mathrm{~g} / \mathrm{s}$, seen in Figure 8. The second mode disturbances continue to experience the largest amplification, so it expected that the most-amplified frequencies will follow the boundary layer thickness. During the injection, the boundary thickness increases from the injection gas, thus resulting in drop in the frequencies being amplified. After the injection, the boundary layer thickness decreases until resuming its natural growth. This change in slope of the boundary layer thickness allows for a band of frequencies to be continuously amplified for a greater length than in the smooth cone case. Due to this flow feature, the recovery distance is the dominant factor that could cause transition for all simulations after the flow has absorbed the disturbance of the injection. Also visible on the stability diagram are amplification of higher modes of disturbances. 


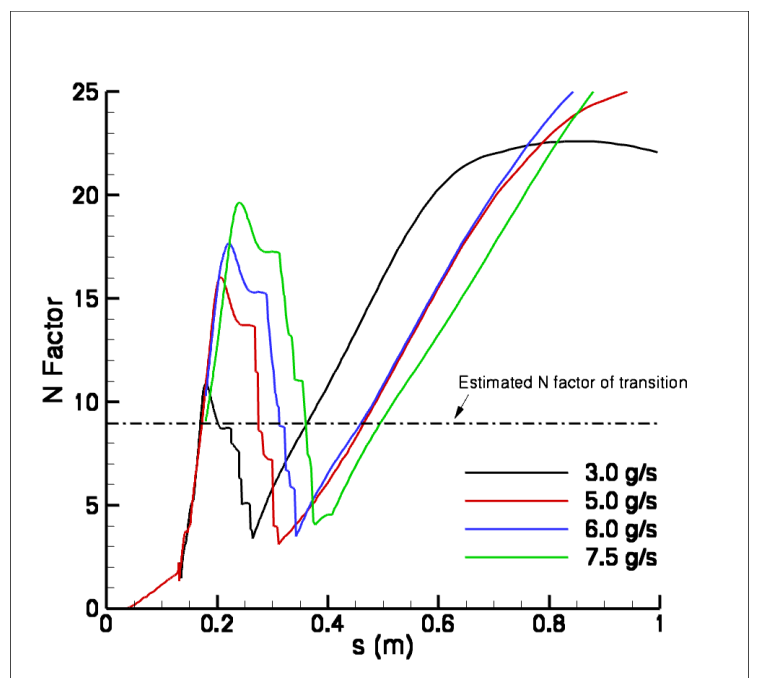

a) Low Mass Flux Cases

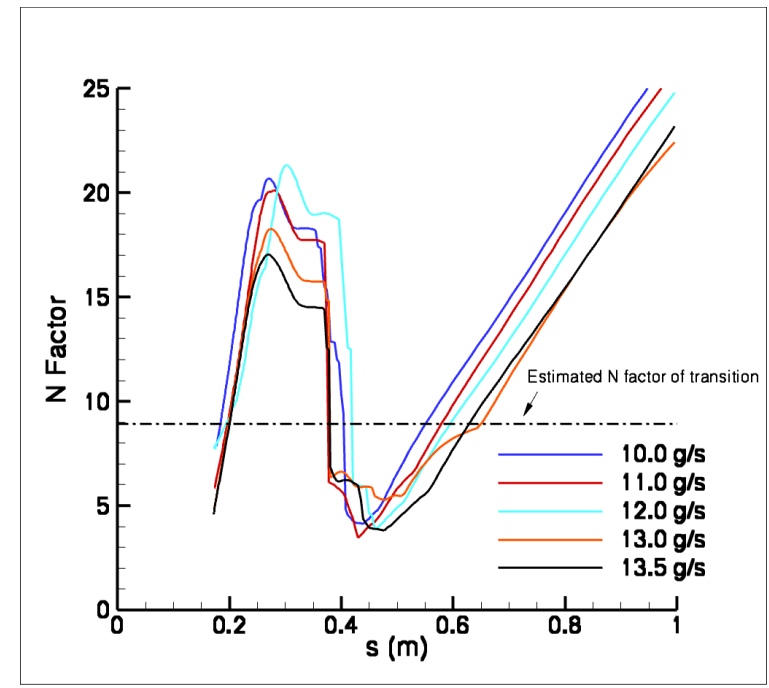

b) High Mass Flux Cases

Figure 7. These plots show the results from the stability analyses of cold carbon dioxide injection. The low mass flux cases are shown in a). The high mass flux cases are shown in b). All cases reach an $\mathbf{N}$ factor sufficient to cause transition immediately following the injection region.

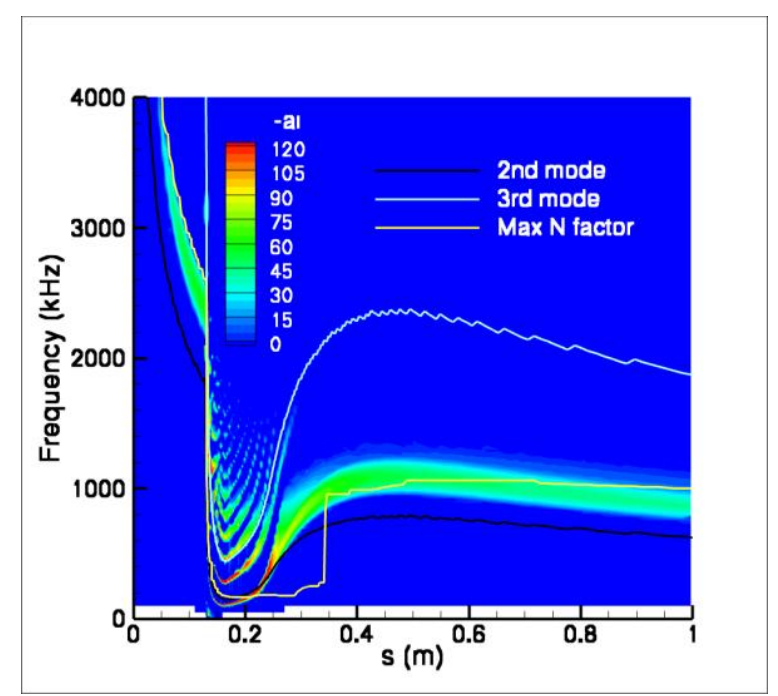

Figure 8. This plot shows the LST stability diagram for the case of cold carbon dioxide injection at a rate of $6.0 \mathrm{~g} / \mathrm{s}$. The sharp drop in amplified frequencies is due the rapid increase in boundary layer thickness. The frequencies rise as the boundary layer recovers from the injection until resuming its natural growth. Also visible is the amplification of higher modes of disturbances.

\section{Alternate Injection Gases}

From previous experiments at the T5 tunnel, it is known that flows involving air and pure nitrogen will transition at a lower Reynolds' number for a given enthalpy than flows involving carbon dioxide. Due to this knowledge, it is expected that injection of carbon dioxide would result in a more stable boundary layer relative to a boundary layer of air or nitrogen. The stability analyses for these cases, seen in Figure 8a, show that this trend does not occur. The similarity of amplification suggests that the momentum of injection is more important in determining the stability when the injection gases are cold. It is easiest to understand the reason for these similarities by inspecting the boundary layer profiles of the case with carbon dioxide injection, seen in Figure 8b. These profiles show that a 
majority of the injected carbon dioxide remains relatively cold. At these low temperatures, the carbon dioxide does not have the ability to absorb disturbance energy with the same capacity that it does at higher temperatures. This agrees with the conclusion of Hornung, that at low enthalpies, carbon dioxide does not have an advantage over air or nitrogen.

\section{Heated Carbon Dioxide Injection}

In order to ensure the effectiveness of the injected carbon dioxide to absorb energy from acoustic disturbances, the gas is heated before injection to a temperature range where the effects of non-equilibrium occur. The carbon dioxide is assumed to be in chemical and thermal equilibrium before injection. For the case of $2500 \mathrm{~K}$ this results in approximately twenty percent, by mass, of the carbon dioxide being dissociated. This implies that fewer molecules are present to absorb energy from disturbances, however, as shown in Figure 2, the carbon dioxide is more efficient around $2000 \mathrm{~K}$ than it is below $1000 \mathrm{~K}$. The stability analyses in Figure 10 show a sufficient increase in stability in the post-injection region to prevent transition until further down the cone. For the case where the injection gas is $1000 \mathrm{~K}$, the $\mathrm{N}$ factor is borderline to transition in the post-injection region; although, with increased temperature this amplification is sufficiently reduced. However, all other cases are expected to transition earlier than the smooth cone case due the amplification that occurs where the boundary layer recovers.

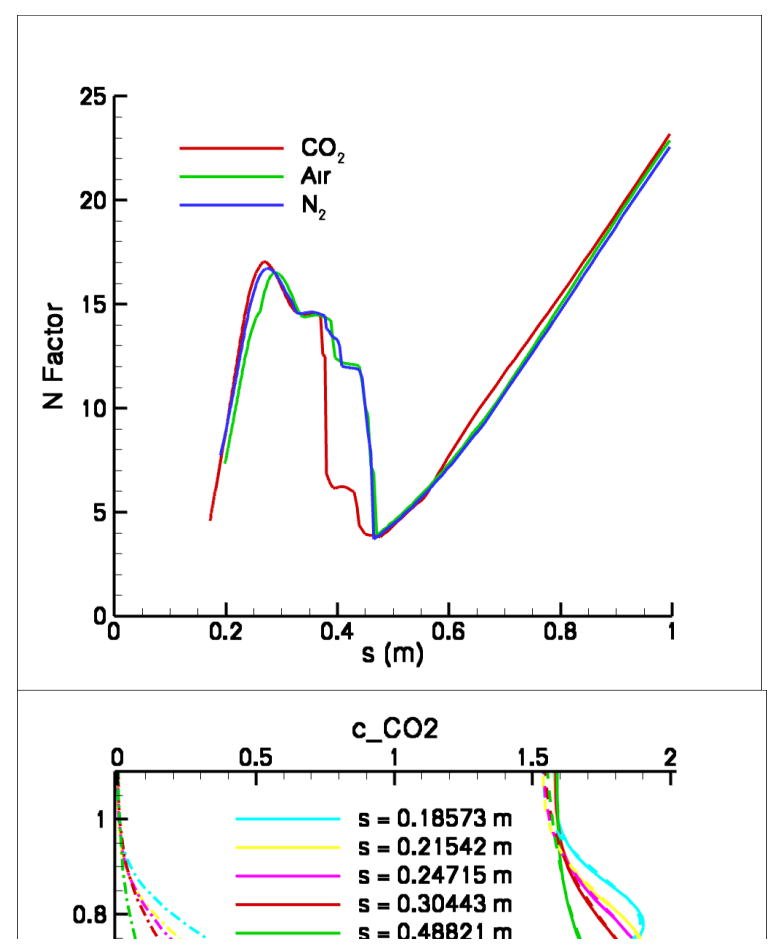




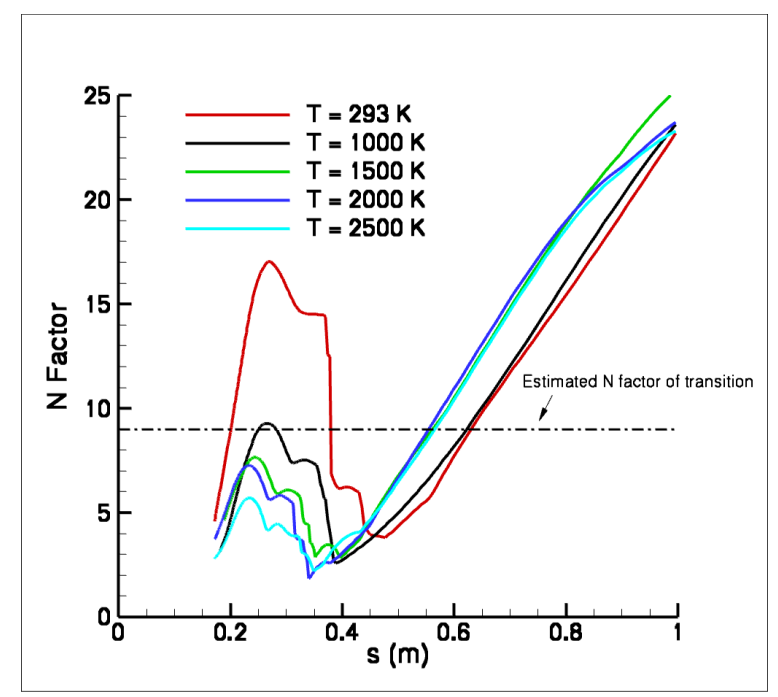

Figure 10. This plot shows the results from the stability analyses of heated carbon dioxide injection. As expected, an increase in temperature of the injected gas further reduces the amplification experienced in the post-injection region.

\section{Theoretical Injection}

Due to the difficulty of injecting a beneficial amount of carbon dioxide over a short interval, the theoretical injection cases have a transpiration interval from $10 \mathrm{~cm}$ to $90 \mathrm{~cm}$ from the nose of the cone. The test body is also a five-degree-half-angle cone. The injection profile is more gradual and is based on a wall-suction model suggested by Malik $^{15}$ and further investigated by Johnson et. al. ${ }^{16}$. The mass flux through the wall is specified by free-stream properties and a dimensionless wall blowing parameter, $\mathrm{f}_{\mathrm{w}}$, seen in Eq. (1).

$$
f_{w}=\frac{\sqrt{2 \operatorname{Re}_{x}} \rho_{w} v_{w}}{\rho_{e} v_{e}}
$$

In this equation, $\mathrm{Re}_{\mathrm{x}}$ is the Reynolds' number based on the surface distance along the cone, $\rho$ is the fluid density, $\mathrm{v}$ is the fluid velocity, the subscript e denotes boundary layer edge properties, and the subscript w denotes wall properties. For these cases the parameter $f_{w}$ is held constant, making the mass flux vary along the length of the cone. Using this model, the dominant factors of rapid boundary layer thickness increase and also the boundary layer recovery seen in the previous cases have been eliminated. It should now be possible to see the effects of nonequilibrium processes that support previous work.

The grid for these simulations is composed of a structured set of quadrilaterals. The wall-normal direction has 300 cells with the spacing at the wall set such that the $y^{+}$value is less than one and the maximum growth rate is 2.7 percent. The wall-tangential direction has 1000 cells, which are clustered to the nose of the cone and have a maximum growth rate of 4.4 percent.

The free-stream consists of a partially dissociated mixture of nitrogen, with the properties contained in Table 2. The free-stream Mach number is 6.5 and the wall condition is isothermal with a temperature of $293 \mathrm{~K}$. Six cases of cold carbon dioxide are run with $\mathrm{f}_{\mathrm{w}}$ ranging from 0.05 to 0.6 . Nitrogen is tested in three cases as the injection gas with $\mathrm{f}_{\mathrm{w}}$ ranging from 0.05 to 0.2 and air is also tested with $\mathrm{f}_{\mathrm{w}}$ set to 0.1 . Finally, the effect of pre-heating the carbon dioxide to a temperature of $1000 \mathrm{~K}$ is also tested in four cases with $\mathrm{f}_{\mathrm{w}}$ ranging from 0.1 to 0.4 . A summary of these test cases is included in the appendix in Table 5. 
Table 2. Free-stream Conditions Used for the Theoretical Injection Simulations.

\begin{tabular}{|c|c|c|c|}
\hline \multicolumn{2}{|c|}{ Stagnation Conditions } & \multicolumn{2}{c|}{ Free-stream Conditions } \\
\hline Pressure (MPa) & 55.0 & Density $\left(\mathrm{kg} / \mathrm{m}^{3}\right)$ & 0.051855 \\
\hline Temperature (K) & 6958 & Temperature $(\mathrm{K})$ & 925.5 \\
\hline Entalpy (MJ/kg) & 9.39 & Velocity $(\mathrm{m} / \mathrm{s})$ & 4039.7 \\
\hline
\end{tabular}

\section{A. Smooth Cone}

A cone without injection is also tested and the stability analysis results in a similar curve as the previous set of free-stream condition, shown in Figure 11a. Using the same critical $\mathrm{N}$ factor as the previous cases, transition is expected to occur near $66 \mathrm{~cm}$ from the nose of the cone. This will serve as the baseline to gauge transition delay.

\section{B. Cold Carbon Dioxide Injection}

The stability results for the cold carbon dioxide injection are as expected and are contained in Figure 11b. Since the injection profile is more gradual than the injection in the T5 tunnel cases, the rapid growth of the boundary layer thickness and rapid amplification is not present for these cases. Initially, the injection of carbon dioxide increases the stability moving transition back to $75 \mathrm{~cm}$. As the mass flux increases the amplification increases. When $\mathrm{f}_{\mathrm{w}}$ is 0.3 , the stabilizing effect of the carbon dioxide is offset by the destabilizing effect of blowing. For the higher values of $f_{w}$ this destabilizing trend continues.

\section{Alternate Injection Gases}

The results from the injection of air and nitrogen are as expected. The stability analyses, seen in Figure 12a, show that only a small difference exists between the injection of air and nitrogen and that blowing for either of these two gases only destabilizes the boundary layer. These qualities suggest that the enthalpy of the free-stream is too low to take advantage of the non-equilibrium effects for these gases and are in agreement with the previous experiments at $\mathrm{T} 5$.

\section{Heated Carbon Dioxide Injection}

Pre-heating the injected carbon dioxide has a similar effect as in the T5 tunnel cases. The stability analyses, seen in Figure 12b, show transition delay for all cases tested with heated carbon dioxide. In the cold injection cases, the carbon dioxide needs some distance along the cone to absorb heat to be in a temperature range where it will more effectively absorb energy from acoustic disturbances. When the carbon dioxide is pre-heated, it starts absorbing disturbance energy earlier, thus resulting in less amplification over the length of the cone. Using a critical $\mathrm{N}$ factor of 9.47, the estimated transition location can be measured. A summary of these locations for all of the theoretical cases tested is plotted in Figure 13. 

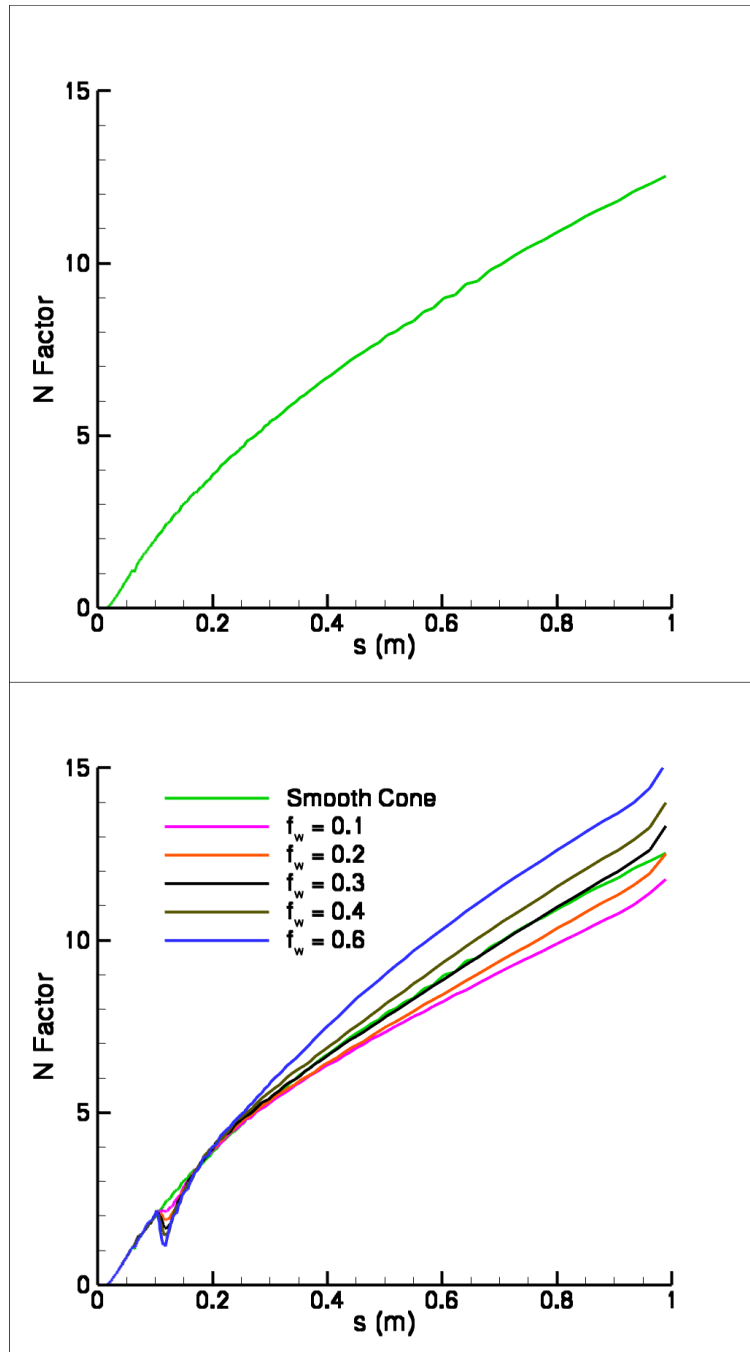

a) Smooth Cone with $\mathbf{N}_{2}$ free-stream

b) $\mathrm{N}$ Factors for Cold $\mathrm{CO}_{2}$ Injection

Figure 11. These plots show the stability analysis results for the smooth cone, a), and cold carbon dioxide injection, $b$ ), with a free-stream composed of nitrogen. The case with $f_{w}$ set to 0.1 shows the maximum transition delay. Cases with high mass flux result in more amplification. 

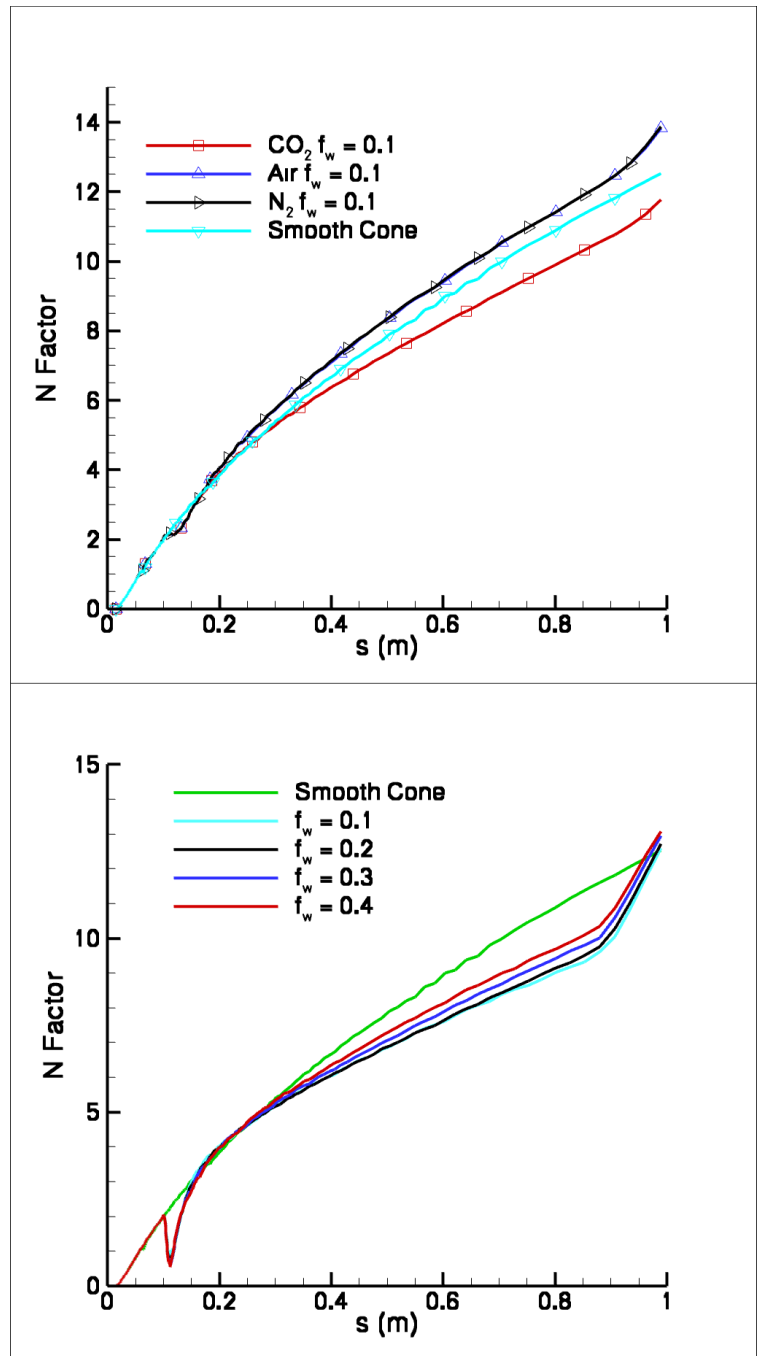

a) $\mathbf{N}$ factors for Alternate Injection Gases $\quad$ b) $\mathbf{N}$ Factors for Heated Carbon Dioxide Injection Figure 12. These plots show the stability analysis results for select cases of alternate gas injection, a), and heated carbon dioxide injection, b). Transition delay does not occur for the alternate gases, while the delay is increased for the heated carbon dioxide cases. 


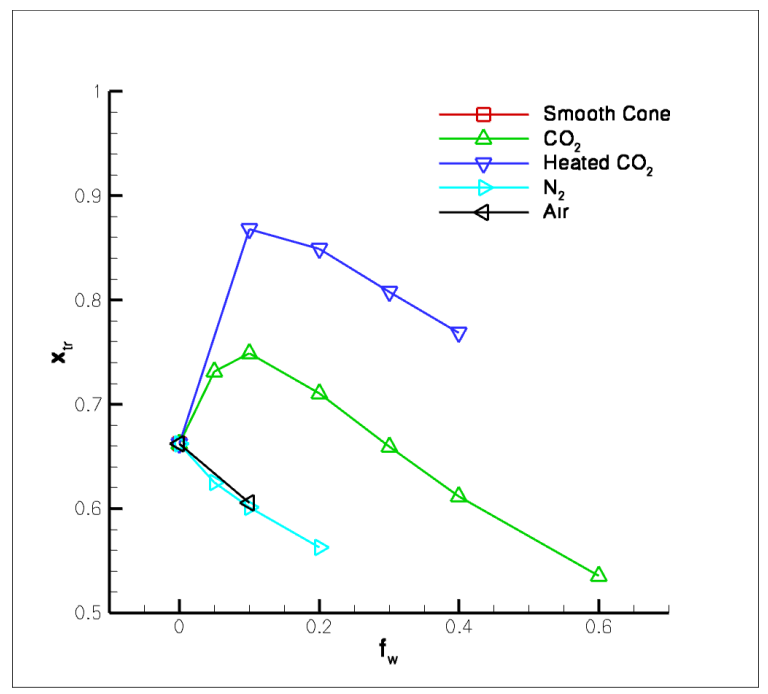

Figure 13. This plot is a summary of the transition locations for the theoretical injection cases based on the critical $\mathbf{N}$ factor of 9.47. The injection of carbon dioxide shows an optimum amount of mass injected.

\section{Effects of Relaxation Processes}

A unique feature of computational analysis is the ability to freeze both the chemical and vibrational modes of disturbance energy absorption. The effect on boundary layer transition delay of each of these modes can then be assessed. The freezing of each relaxation process is done in PSE-chem analysis, while the mean flow has both vibration and chemistry active. This test is performed for the injection of cold carbon dioxide, heated carbon dioxide and nitrogen all with $\mathrm{f}_{\mathrm{w}}$ set to 0.1 . As seen in Figure 14, both the full relaxation and vibrational relaxation from the injection of cold carbon dioxide follow the same curve, indicating that the vibrational relaxation is responsible for absorbing energy from disturbances. To verify this, a stability analysis with only chemical relaxation is compared to one with no relaxation processes active and is found to be identical. For this temperature range, the vibrational mode is expected to be the dominant mechanism for reducing the amplification of acoustic disturbances, as discussed in Fujii and Hornung ${ }^{4}$. It is interesting that a reduction of amplification still occurs when non-equilibrium effects in the disturbances are frozen. These data suggest that the gain in stability is also due to other aspects of injecting carbon dioxide. For the heated carbon dioxide case, a similar trend occurs with the only difference being the amplitude with which the $\mathrm{N}$ factors are increased without vibrational relaxation. The case with nitrogen shows no difference in amplification, verifying that the non-equilibrium in the disturbances has no effect for nitrogen for this temperature range. 


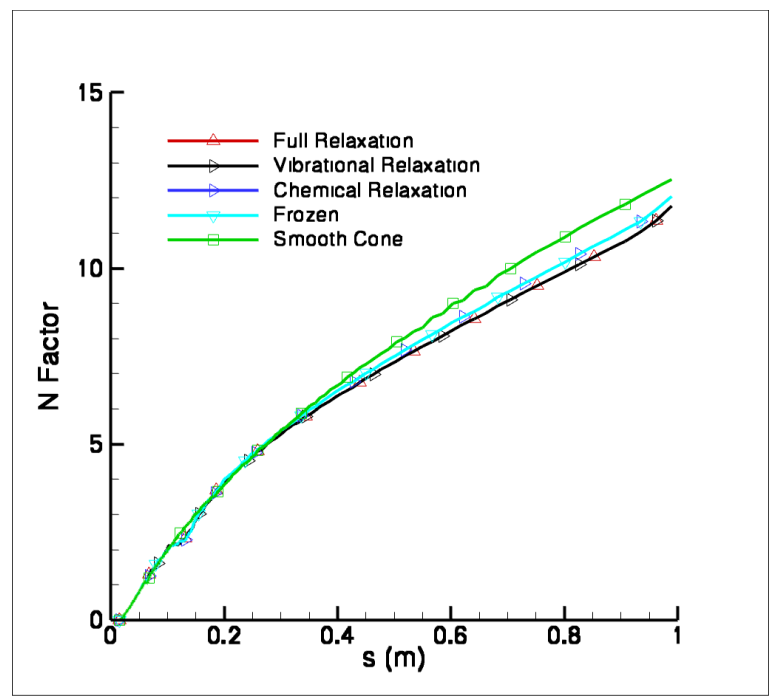

Figure 14. This plot shows the effect of non-equilibrium processes on the disturbances for cold carbon dioxide with $\mathbf{f}_{\mathrm{w}}$ set to 0.1 . It is interesting that a reduction in amplification still occurs despite these processes being frozen.

\section{Conclusion}

This study tested the feasibility of injecting carbon dioxide as a means of boundary layer transition delay through the use of numerical simulations for two different test bodies. The first test case was a five-degree-half-angle cone with a four $\mathrm{cm}$ interval of transpiration and was modeled after an experimental test body that will be used in the T5 Shock Tunnel at Caltech. The simulations predicted that transition will occur immediately following the injection. The test cases with air and nitrogen as the test gas suggested the momentum of the injection plays a dominant role in the amount of amplification seen immediately downstream due to the inefficient heating of the injected carbon dioxide. To remedy this, the injected carbon dioxide was pre-heated, resulting in a reduction of amplification in the post-injection region. Despite this reduction, the stability analyses still predicted transition to occur earlier on the cone as compared to a case without injection.

To isolate the effects of non-equilibrium processes on the disturbances, a second case involving a similar cone with a longer transpiration interval was tested. For this case, the stability analyses predicted a window of carbon dioxide injection resulting in a reduction of amplification and thus a delay in boundary layer transition. The cold carbon dioxide injected experienced sufficient heating to cause transition delay. Pre-heating the carbon dioxide showed a further delay in transition. The analyses further indicated an optimum amount of carbon dioxide injection as a balance between the stabilizing effects of carbon dioxide and the destabilizing effect of wall-blowing. Testing on the injection of air and nitrogen predicted no transition delay would occur and an increase in wall-blowing simply causes more amplification.

Finally, the effect of specific non-equilibrium processes was tested in order to investigate the contribution of each of these modes. Stability analyses predicted that vibrational relaxation contributes to damping acoustic disturbances, while chemical relaxation does not for the enthalpy range tested.

Further work includes experimental testing of the first test body in the T5 Shock Tunnel. This work will be very important to validate all of the models used to simulate these experiments, as well as the application of stability analysis for this type of flow. Should the results of these analyses prove to be accurate; an alternative means of injection will be explored.

\section{Acknowledgements}

Insert acknowledgements 


\section{References}

${ }^{1}$ Leyva, I. A., Laurence, S., Beierholm, A. W., Hornung, H.G., Wagnild, R. W., and Candler, G. V., "Transition Delay in Hypervelocity Boundary Layers by Means of CO2/acoustic Instability Interactions," AIAA Paper 2009-1287, January 2009.

${ }^{2}$ Hornung, H. G., Adam, P. H., Germain, P., Fujii, K., and Rasheed, A., "On Transition and Transition Control in Hypervelocity Flows," Proceedings of the Ninth Asian Congress of Fluid Mechanics, Isfahan, Iran, 2002.

${ }^{3}$ Johnson, H. B., Seipp, T. G., and Candler, G. V., "Numerical Study of Hypersonic Reacting Boundary Layer Transition on Cones," Physics of Fluids, vol. 10, No. 10, October 1998.

${ }^{4}$ Fujii, K., and Hornung, H. G., "Experimental Investigation of High Enthalpy Effects on Attachment-line Boundary Layer Transition," AIAA, vol. 41, no. 7, July 2003.

${ }^{5}$ Leyva, I. A., Jewell, J. S., Laurence, S., Hornung, H. G., Shepherd, J. E., "On the impact of injection schemes on transition in hypersonic boundary layers," AIAA Paper 2009-7204, October 2009.

${ }^{6}$ Wright, M. J., Candler, G. V., and Bose, D., "A Data-Parallel Line-Relaxation Method for the Navier-Stokes Equations," AIAA Paper 97-2046CP, June 1997.

${ }^{7}$ Johnson, H. B., and Candler, G. V., "Hypersonic Boundary Layer Stability Analysis Using PSE-Chem," AIAA Paper 20055023, June 2005.

${ }^{8}$ Johnson, H. B., Candler, G. V., and Wright, M. J., "Boundary Layer Stability Analysis of Mars Science Laboratory Aeroshell," AIAA Paper 2006-0920, January 2006.

${ }^{9}$ Camac, M., "CO2 Relaxation Processes in Shock Waves," Fundamental Phenomena in Hypersonic Flow, Ed. J.G. Hall, Cornell University Press, Ithaca, NY, 1966, pp. 195-215.

${ }^{10}$ Park, C., Howe, J.T., Jaffe, R.J., and Candler, G.V., "Review of Chemical-Kinetic Problems of Future NASA Missions, II: Mars Entries," Journal of Thermophysics and Heat Transfer, Vol. 8, No. 1, 1994, pp. 9-23.

${ }^{11}$ Bose, D. and Candler, G. V. "Thermal Rate Constants of the N2 + O -> NO + N Reaction Using Ab Initio 3a" and 3a' Potential Energy Surfaces,” Journal of Chemical Physics, vol. 104, No. 8, pp. 2825-2833, 1996.

${ }^{12}$ Bose, D. and Candler, G. V. "Thermal Rate Constants of the O2 + N $->$ NO + O Reaction Based on the 2a' and 4a' Potential Energy Surfaces,” Journal of Chemical Physics, vol. 107, No. 16, pp. 6136-6145, 1997.

${ }^{13}$ Park, C., Nonequilibrium Hypersonic Aerodynamics, Wiley, 1990.

${ }^{14}$ McBride, B. J., Zehe, M. J., and Gordon, S. "NASA Glenn Coefficients for Calculating Thermodynamic Properties of Individual Species,” Tech. Rep. 2002-211556, NASA, September 2002.

${ }^{15}$ Malik, M. R., "Prediction and Control of Transition in Supersonic and Hypersonic Boundary Layers," AIAA Journal, vol. 27, no. 11, November 1989, pp. 1487-1493.

${ }^{16}$ Johnson, H. B., Gronvall, J. E., Candler, G. V., "Reacting Hypersonic Boundary Layer Stability with Blowing and Suction,” AIAA Paper 2009-938, January 2009.

\section{Appendix}

\section{Table 3. Reaction set used for the CFD simulations}

$$
\begin{aligned}
& \mathrm{CO}_{2}+M \Leftrightarrow C O+O+M \\
& \mathrm{~N}_{2}+M \Leftrightarrow N+N+M \\
& \mathrm{O}_{2}+M \Leftrightarrow O+O+M \\
& N O+M \Leftrightarrow N+O+M \\
& N_{2}+O \Leftrightarrow N O+N \\
& O_{2}+N \Leftrightarrow N O+O \\
& C O_{2}+O \Leftrightarrow O_{2}+C O
\end{aligned}
$$


Table 4. Summary of cases tested for the T5 tunnel test body

\begin{tabular}{|c|c|c|c|}
\hline Case & Injection Gas & Temperature (K) & Mass Flux (g/s) \\
\hline 1 & $\mathrm{CO}_{2}$ & 293 & 3.0 \\
\hline 2 & $\mathrm{CO}_{2}$ & 293 & 5.0 \\
\hline 3 & $\mathrm{CO}_{2}$ & 293 & 6.0 \\
\hline 4 & $\mathrm{CO}_{2}$ & 293 & 7.5 \\
\hline 5 & $\mathrm{CO}_{2}$ & 293 & 10.0 \\
\hline 6 & $\mathrm{CO}_{2}$ & 293 & 11.0 \\
\hline 7 & $\mathrm{CO}_{2}$ & 293 & 12.0 \\
\hline 8 & $\mathrm{CO}_{2}$ & 293 & 13.0 \\
\hline 9 & $\mathrm{CO}_{2}$ & 293 & 13.5 \\
\hline 10 & $\mathrm{Air}$ & 293 & 10.93 \\
\hline 11 & $\mathrm{~N}_{2}$ & 293 & 10.77 \\
\hline 12 & $\mathrm{CO}_{2}$ & 1000 & 9.43 \\
\hline 13 & $\mathrm{CO}_{2}$ & 1500 & 7.7 \\
\hline 14 & $\mathrm{CO}_{2}$ & 2000 & 6.9 \\
\hline 15 & $\mathrm{CO}_{2}$ & 2500 & 6.3 \\
\hline
\end{tabular}

Table 5. Summary of cases tested for the theoretical test body

\begin{tabular}{|c|c|c|c|}
\hline Case & Injection Gas & Temperature $(\mathrm{K})$ & $\mathrm{f}_{\mathrm{w}}$ \\
\hline 1 & $\mathrm{CO}_{2}$ & 293 & 0.05 \\
\hline 2 & $\mathrm{CO}_{2}$ & 293 & 0.1 \\
\hline 3 & $\mathrm{CO}_{2}$ & 293 & 0.2 \\
\hline 4 & $\mathrm{CO}_{2}$ & 293 & 0.3 \\
\hline 5 & $\mathrm{CO}_{2}$ & 293 & 0.4 \\
\hline 6 & $\mathrm{CO}_{2}$ & 293 & 0.6 \\
\hline 7 & $\mathrm{~N}_{2}$ & 293 & 0.05 \\
\hline 8 & $\mathrm{~N}_{2}$ & 293 & 0.1 \\
\hline 9 & $\mathrm{~N}_{2}$ & 293 & 0.2 \\
\hline 10 & $\mathrm{Air}$ & 293 & 0.1 \\
\hline 11 & $\mathrm{CO}_{2}$ & 1000 & 0.05 \\
\hline 12 & $\mathrm{CO}_{2}$ & 1000 & 0.1 \\
\hline 13 & $\mathrm{CO}_{2}$ & 1000 & 0.2 \\
\hline 14 & $\mathrm{CO}_{2}$ & 1000 & 0.3 \\
\hline 15 & $\mathrm{CO}_{2}$ & 1000 & 0.4 \\
\hline
\end{tabular}

